Anderer, Pia; Bauer, Nikolas; Dumont, Ulrich; Heimerl, Stephan; Ruprecht, Albrecht; Wolf-Schumann, Ulrich

\title{
Das Wasserkraftpotenzial in Deutschland
}

Zur Verfügung gestellt in Kooperation mit / Provided in Cooperation with:

Technische Universität Dresden, Institut für Wasserbau und technische Hydromechanik

Verfügbar unter / Available at:

https://hdl.handle.net/20.500.11970/103588

Vorgeschlagene Zitierweise / Suggested citation:

Anderer, Pia; Bauer, Nikolas; Dumont, Ulrich; Heimerl, Stephan; Ruprecht, Albrecht; Wolf-Schumann, Ulrich (2011): Das Wasserkraftpotenzial in Deutschland. In: Technische Universität Dresden, Institut für Wasserbau und technische Hydromechanik (Hg.): Wasserkraft mehr Wirkungsgrad + mehr Ökologie = mehr Zukunft. Dresdner Wasserbauliche Mitteilungen 45. Dresden: Technische Universität Dresden, Institut für Wasserbau und technische Hydromechanik. S. 19-28. 


\title{
Das Wasserkraftpotenzial in Deutschland
}

\author{
Pia Anderer, Nikolaus Bauer, Ulrich Dumont, Stephan Heimerl, \\ Albert Ruprecht, und Ulrich Wolf-Schumann
}

\begin{abstract}
Deutschland hat sich zum Ziel gesetzt, 2030 einen Deckungsanteil der erneuerbaren Energien am gesamten Stromverbrauch von 45 \% zu erreichen. In 2009 stellten sie insgesamt einen Anteil von etwa $16 \%$ bereit, die Wasserkraft hatte daran einen Anteil von ca. $20 \%$ [1]. Das zusätzlich ausbaubare Wasserkraftpotenzial wurde auf der Basis des Linienpotenzials unter Berücksichtigung gewisser Restriktionen bundesweit ermittelt. Bei Anlagen der Leistung $\geq 1$ MW erfolgte eine Standort spezifische Untersuchung.
\end{abstract}

Germany has set a goal for the year 2030 to cover $45 \%$ of its electric power consumption through renewable energies. In 2009 about $16 \%$ of electricity were provided by renewable energy sources, $20 \%$ of which came from hydro power [1]. The additionally usable hydropower potential throughout Germany was determined, using the consistent method of theoretical line potential calculation. For installations $\geq 1 \mathrm{MW}$ the supplementary hydro power potential was investigate on a local basis.

\section{Einleitung}

Im Auftrag des Bundesministeriums für Umwelt, Naturschutz und Reaktorsicherheit (BMU) wurde auf der Grundlage einer für ganz Deutschland einheitlichen Methode das zusätzlich ausbaubare Wasserkraftpotenzial ermittelt. Als Potenzial wird dabei wie in der Physik üblich die Fähigkeit definiert, Arbeit zu verrichten. Die Einheit ist daher die einer Arbeit bzw. Energie: Wh bzw. kWh, MWh, GWh etc. Das Projekt wurde von einem Expertenbeirat begleitet, an dem Vertreter von Behörden, Wasserkraftbetreibern und Naturschutzverbänden beteiligt waren.

Die seit Beginn des 20. Jahrhunderts erschienenen Veröffentlichungen zum Wasserkraftpotenzial [2] bis [10], basieren im Wesentlichen auf zwei Methoden:

- der Standort-Methode, bei der die Ausbaumöglichkeiten an konkreten Standorten bzw. Wasserkraftanlagen betrachtet werden und 
- der Linienpotenzial-Methode, bei der das natürlich vorhandene, aber nur theoretisch verfügbare Wasserkraftpotenzial aus Abfluss und Höhendifferenzen ermittelt wird.

In dem hier beschriebenen Vorhaben wurden beide Methoden angewendet: mit Hilfe der Standort-Methode wurde das Zubaupotenzial bestehender Wasserkraftanlagen mit $\mathrm{P} \geq 1 \mathrm{MW}$ detailliert untersucht. Aus dem theoretischen Linienpotenzial wurde das technische und das realisierbare Zubau- und Ausbaupotenzial abgeschätzt.

\section{Aktuelle Wasserkraftnutzung}

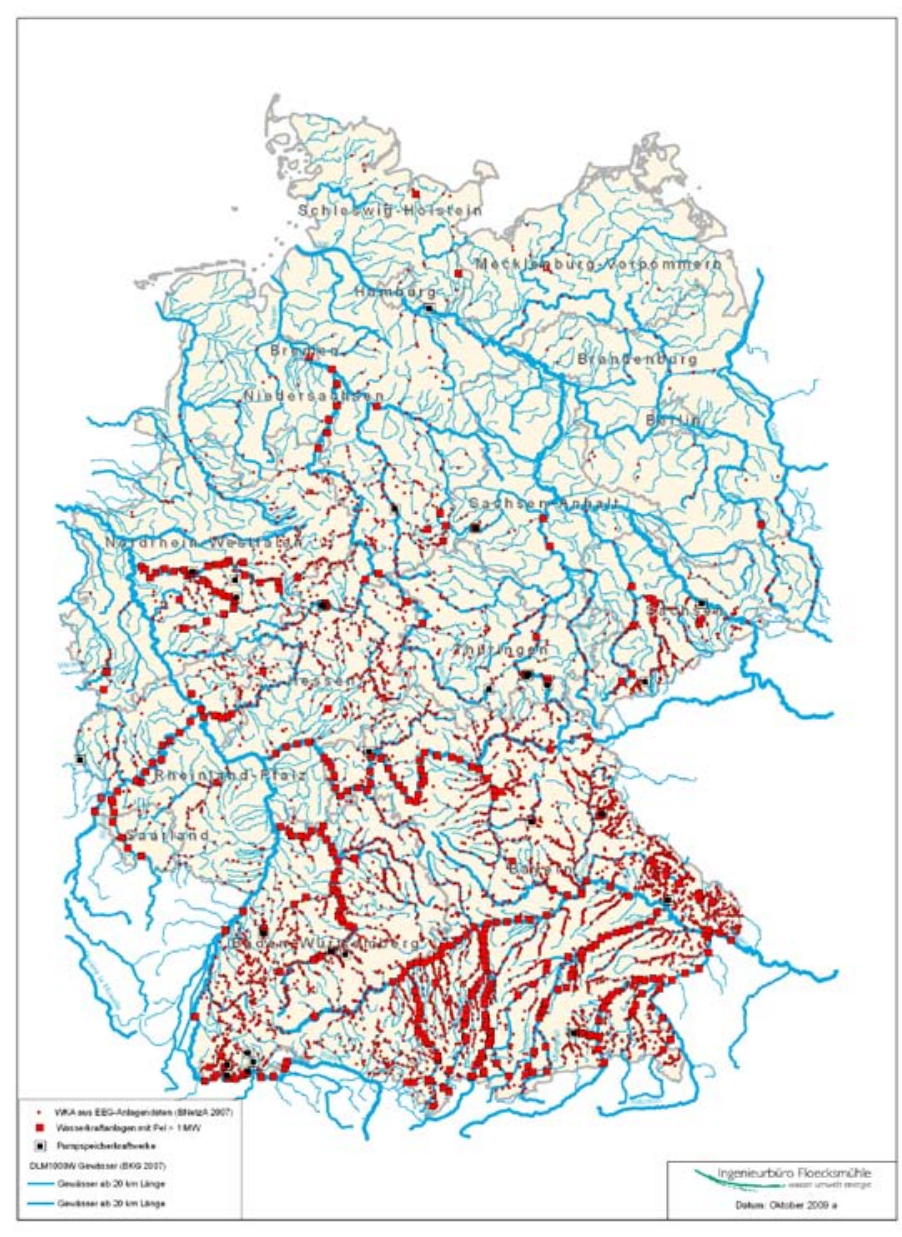

Die Wasserkraft gilt in der Bundesrepublik Deutschland als relativ gut ausgebaut. An den meisten potentiellen Standorten mit einer installierten Leistung $\geq 1 \mathrm{MW}$ sind bereits Wasserkraftanlagen installiert. Die Stromerzeugung aus Wasserkraft unterlag in den letzten Jahrzehnten natürlichen jährlichen Schwankungen von über $\pm 15 \%$. Daher muss beim Vergleich veröffentlichter Zahlen auf Zeitangaben und darüber hinaus auf die Art der Berücksichtigung Grenzkraftwerken geachtet werden.

In der vorliegenden Untersuchung wurden etwa 6.700 in Betrieb befindliche WKA ermittelt (Abbildung 1).

Abbildung 1 Wasserkraftanlagen in Betrieb [11]

Darüber hinaus existieren schätzungsweise 600 bis 900 Wasserkraftanlagen, die für die Eigenversorgung arbeiten. Die installierte Leistung der ermittelten WKA (ohne Pumpspeicherkraftwerke) beträgt etwa 4,05 GW, die durchschnittlich erzeugte Jahresarbeit 20,9 TWh (inklusive Anteil aus natürlichem Zufluss von Pumpspeicherkraftwerken). In diesen Angaben ist für Grenzkraftwerke nur der 
deutsche Anteil berücksichtigt. Von den 406 Wasserkraftanlagen der Leistung P $\geq 1 \mathrm{MW}$ wurde $84 \%$ der gesamten Jahresarbeit bereit gestellt. Den größten Beitrag zum genutzten Potenzial liefern die Länder Bayern und BadenWürttemberg, in denen zusammen etwa 80 \% der Jahresarbeit erzeugt wird (Abbildung 2, logarithmische Auftragung).

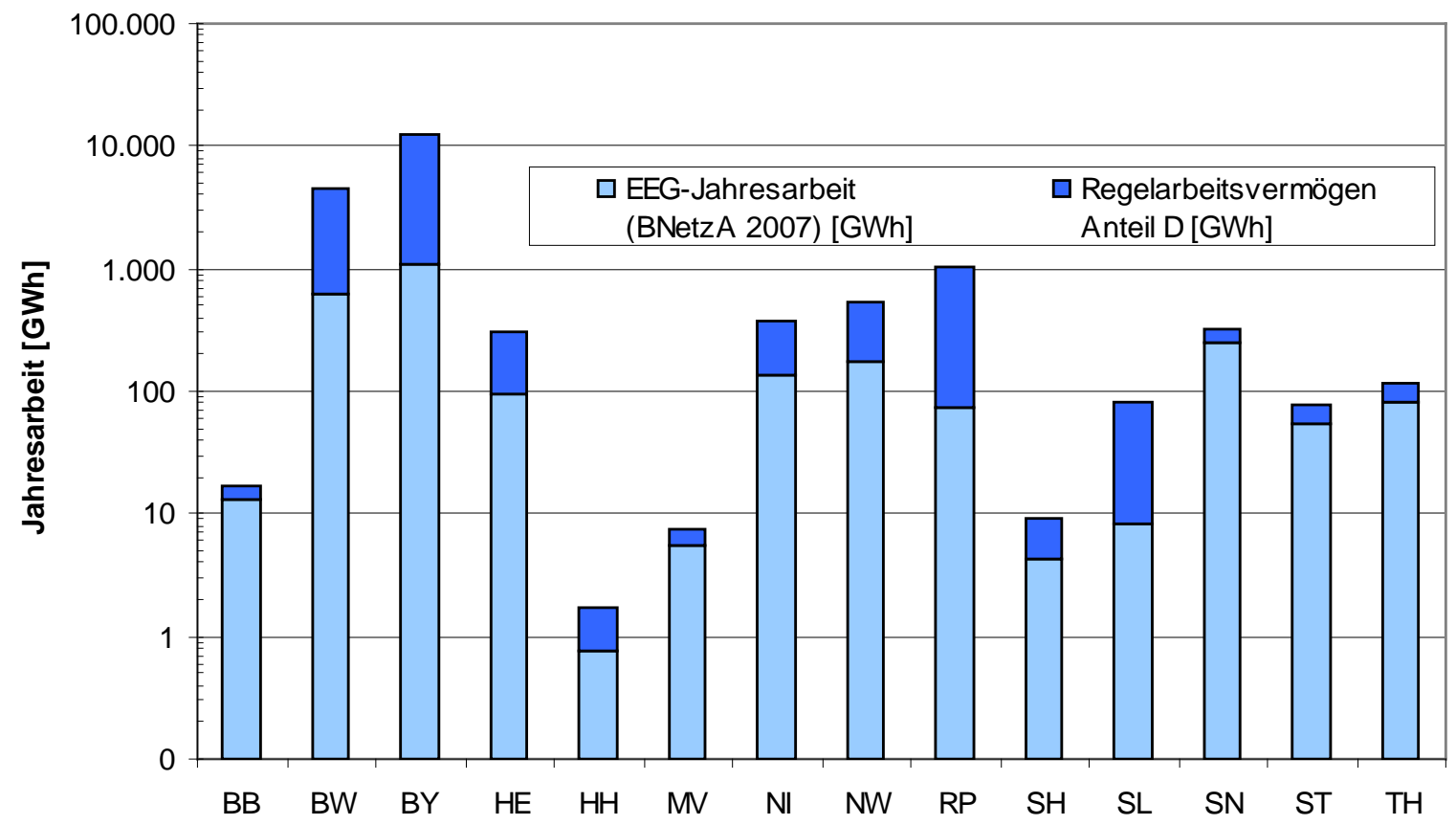

Abbildung 2 Jahresarbeit deutscher Wasserkraftanlagen, Stand 2007; dargestellt ist die EEG vergütete Strommenge für WKA mit $\mathrm{P}<1 \mathrm{MW}$ und das Regelarbeitsvermögen (RAV) für WKA mit $\mathrm{P} \geq 1 \mathrm{MW}$

\section{Das theoretische Linienpotenzial deutscher Gewässer}

Auf der Grundlage digital vorliegender Daten zur Geländemorphologie und zu den Abflüssen wurden die Linienleistung und das theoretische Linienpotenzial in einem Geografischen Informationssystem (GIS) für ganz Deutschland berechnet [12]. Das theoretische Linienpotenzial beträgt für alle Gewässer mit einem Einzugsgebiete größer $10 \mathrm{~km}^{2}$ (Grenzflüsse anteilig berücksichtigt):

$\mathrm{E}_{\mathrm{L}} \quad=92,6 \mathrm{TWh}$.

Das Linienpotenzial kann nie vollständig genutzt werden, denn

- Fließverluste der Gewässer (u. a. Turbulenzen, Geschiebetransport) und Anlagenverluste reduzieren das Linienpotenzial auf das technische Rohpotenzial. 
- Turbinen können nicht den gesamten jährlichen Abfluss verarbeiten. Unter Berücksichtigung der realen Nutzungsmöglichkeiten ergibt sich das technische Potenzial.

Die genannten Verluste wurden für große Gewässer, an denen sich vorwiegend WKA der Leistung $\geq \_1 \mathrm{MW}$ befinden und für mittelgroße bis kleine Gewässer spezifisch bestimmt.

\section{Das technische Potenzial}

Als technisches Potenzial $\mathrm{E}_{\text {tech }}$ stellt hier den maximalen Wert dar, der unter realen Bedingungen allerdings bei durchgehendem Einstau der Gewässer genutzt werden kann. Bei der Herleitung waren Annahmen und Näherungen bezüglich der Anlagenwirkungsgrade, Jahresvolllaststunden und Fließverluste erforderlich. Letztere gingen dabei als Spanne in die Berechnungen ein.

Die Untersuchung zeigte, dass für große Gewässer maximal etwa $60 \%$ des Linienpotenzials technisch genutzt werden kann, während bei mittelgroßen bis kleinen Gewässern dieser Prozentsatz je nach angenommenem Ausbaugrad auf weniger als $20 \%$ zurückgeht (Abbildung 3). Das gesamte technische Potenzial in Deutschland wurde ermittelt zu:

$\mathrm{E}_{\text {tech }}=33,2$ bis $42,1 \mathrm{TWh}$.

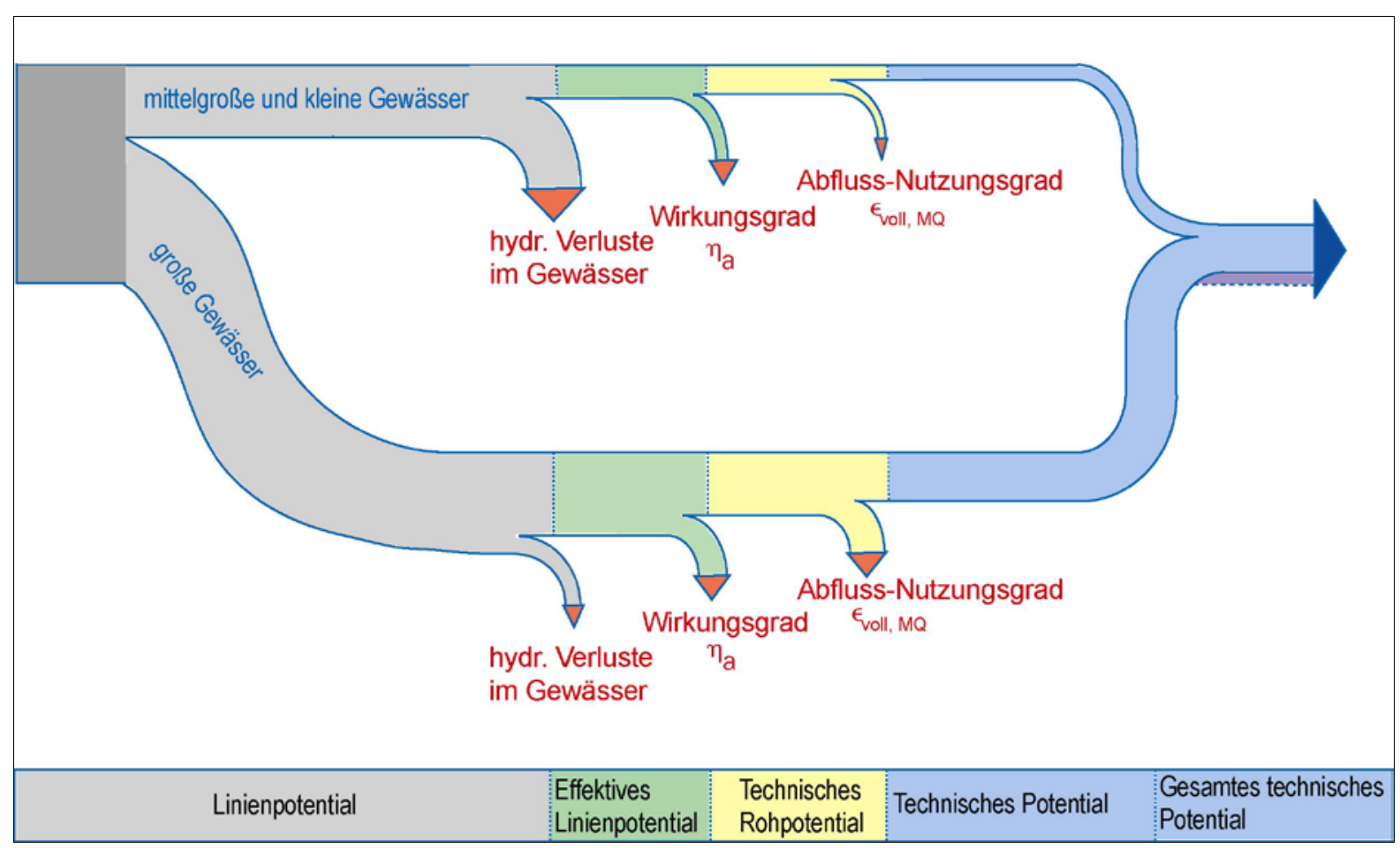

Abbildung 3 Vom Linienpotenzial zum technischen Potenzial, differenziert für große sowie für mittelgroße und kleine Gewässer. 


\section{Das Zubaupotenzial an großen Gewässern}

Das nicht genutzte technische Potenzial ergibt sich für die großen Gewässer nach Abzug des bereits genutzten Potenzials von 17,5 TWh zu 10,9 bis 18,5 TWh (im Mittel 14,7 TWh, Abbildung 4). Neben dem Potenzial größerer zusammenhängender „frei fließender Strecken“ an Rhein, Elbe, Oder und Donau (im Mittel 10,7 TWh), setzt es sich aus dem Zubaupotenzial von 0,1 bis 0,12 TWh an ungenutzten Querbauwerken (mit Möglichkeit zum Bau von WKA mit $\mathrm{P} \geq 1 \mathrm{MW}$ ), dem technischen Verbesserungspotenzial an bestehenden Standorten mit $\mathrm{P} \geq 1 \mathrm{MW}$ (im Mittel 2,55 TWh) und einem technischen Restpotenzial von 1,3 TWh zusammen.

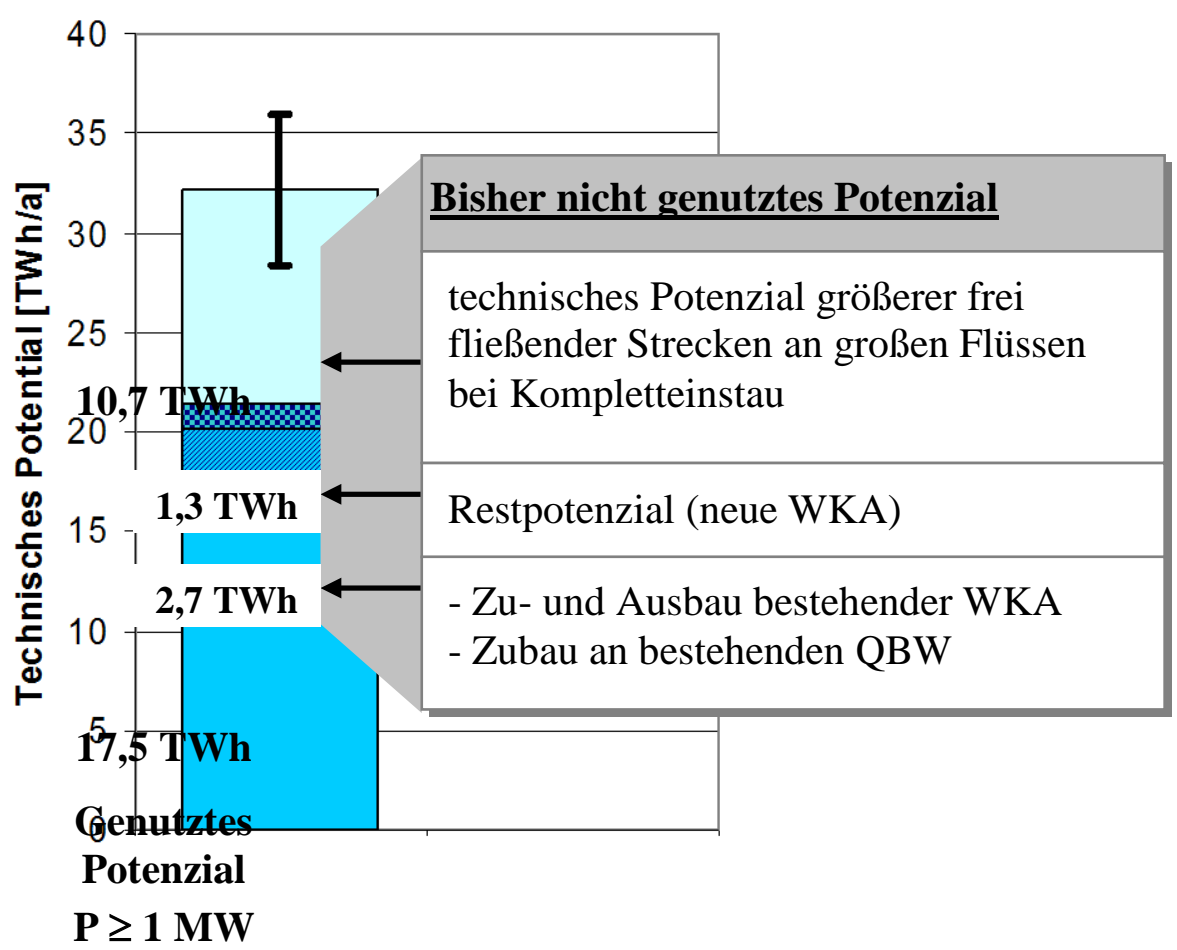

Abbildung 4 Aufteilung des technischen Potenzials großer Gewässer

Das technische Verbesserungspotenzial bestehender WKA mit $\mathrm{P} \geq 1 \mathrm{MW}$ konnte basierend auf den ermittelten Hauptdaten durch eine Standort spezifische Analyse bestimmt werden [14]. Es setzt sich zusammen aus zusätzlichen Potenzialen durch Erhöhung des Anlagenwirkungsgrades (1.120 GWh) und durch Erhöhung des Ausbaugrades (1.430 GWh).

Das so berechnete zusätzliche Potenzial bedeutet eine Erhöhung der durch große Laufwasserkraftanlagen gewonnenen Energie um fast 15 \%. Der Großteil des 
verfügbaren Ausbaupotenzials (fast $80 \%$ ) konzentriert sich dabei ebenso wie das bereits genutzte Potenzial auf neun große Flüsse (Abbildung 5).

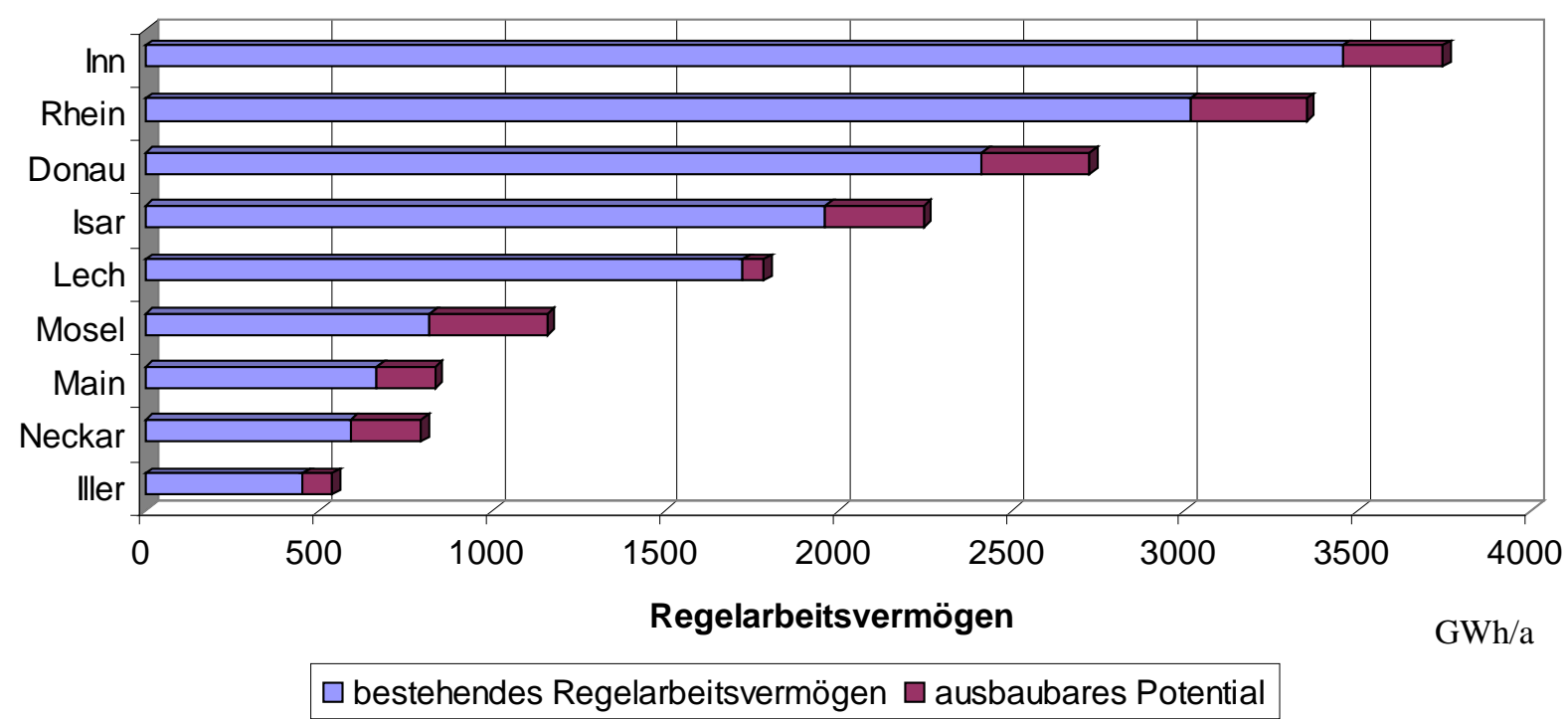

Abbildung 5 RAV in GWh/a sowie ermitteltes Zubaupotenzial bei Erhöhung von Anlagenwirkungsgrad und Ausbaugrad, WKA P $\geq 1 \mathrm{MW}$ an neun großen Flüssen

\section{Das realisierbare Zubaupotenzial an großen Gewässern}

Da sich das technische Verbesserungspotenzial und das Zubaupotenzial an bestehenden Querbauwerken befindet, kann erwartet werden, dass dieses Zubaupotenzial von ca. 2,7 TWh realisierbar ist. Die Nutzung des Restpotenzials von ca. 1,3 TWh wäre dagegen nur bei durchgehendem Aufstau“ und dem Neubau von optimierten Wasserkraftanlagen außerhalb der „größeren frei fließenden Gewässerstrecken realisierbar. Ein derartiger Ausbau ist vor dem Hintergrund ökologischer Anforderungen sowie aufgrund unterschiedlicher Nutzungsansprüche, wie Besiedlung, Hochwasserschutz, Infrastruktur etc., als schwierig einzuschätzen.

\section{Das Zubaupotenzial für mittelgroße und kleine Gewässer}

Zur Zeit werden vom technischen Potenzial, das an mittelgroßen und kleinen Gewässern 4,8 bis 6,1 TWh beträgt, etwa 3,4 TWh genutzt (Abbildung 6). Das verbleibende, technische Zubaupotenzial von 1,4 bis 2,7 TWh (im Mittel 2,1 TWh) wird durch folgende Bedingungen verringert [12]:

- Erhalt frei fließender Gewässerstrecken aufgrund nutzungsbedingter Restriktionen durch Besiedlung, Landwirtschaft, Hochwasserschutz etc. und Erhalt frei fließender Gewässerstrecken in NATURA-2000- bzw. anderen 
Schutzgebieten. Insgesamt wird dieser Anteil am nicht genutzten technischen Potenzial auf 1 TWh geschätzt.

- Bereitstellung ökologischer Abflüsse, die Bypässe und Fischaufstiegsanlagen bedienen. Sie stellen u. a. bei Ausleitungskraftwerken den Abfluss und die Durchwanderbarkeit im Mutterbett sicher. Diese Potenzialminderung wird auf 0,1 TWh geschätzt.

Das verbleibende technischen Zubaupotenzial von etwa 0,63 bis 1,22 TWh (im Mittel 1 TWh) beinhaltet neben dem Potenzial für den Neubau von Wasserkraftanlagen auch das technische Verbesserungspotenzial an bestehenden WKA z. B. durch Verbesserungen der Wirkungsgrade, der Steuerung, der Rechenreinigung und der Betriebsführung. Dieses wird von vielen Autoren auf etwa $20 \%$ des genutzten Potenzials beziffert und beträgt damit etwa 0,56 TWh.

Für den Neubau von Wasserkraftanlagen verbleibt also die Differenz aus dem technischen Zubaupotenzial und dem technischen Verbesserungspotenzial. Im Mittel beträgt es etwa 0,4 TWh.
0,63 bis 1,22 TWh
- $\quad 0,56 \mathrm{TWh}$
$=0,07$ bis $0,66 \mathrm{TWh}$
Technisches
Zubaupotenzial
Technisches
Verbesserungspotenzial
Technisches
Neubaupotenzial

Insgesamt wäre das technische Zubaupotenzial nutzbar, wenn außerhalb „frei fließender Gewässerstrecken“ und außerhalb der Strecken in NATURA-2000Gebieten an bestehenden Querbauwerken neue Wasserkraftanlagen gebaut würden oder wenn durch den Neubau optimierter Wasserkraftanlagen bestehende Anlagen ersetzt würden. Die Spanne repräsentiert die Unsicherheit bei der Umsetzung des Ausbaus.

\section{Das realisierbare Zusatzpotenzial an mittelgroßen und kleinen Gewässern}

Eine Abschätzung in Analogie zu Untersuchungen aus Rheinland-Pfalz [9] und Nordrhein-Westfalen [10] zeigt, dass mit dem EEG 2009 etwa $60 \%$ des mittleren Zubaupotenzials von ca. 1 TWh, d. h. etwa 0,6 TWh wirtschaftlich realisierbar sind. Dieses technisch-ökonomisch-ökologische Zubaupotenzial entspricht einer Steigerung der Wasserkraftnutzung an mittelgroßen und kleinen Gewässern um etwa $18 \%$. Bei einer Volllaststundenzahl von etwa 4.500 h/a entspricht das einer installierten Leistung von $600 \mathrm{GWh} / 4.500 \mathrm{~h}=133 \mathrm{MW}$. 
Würde der Anteil des enthaltenen Neubaupotenzials von im Mittel 53,2 MW durch Anlagen einer mittleren Leistung von $200 \mathrm{~kW}$ realisiert, entspräche das dem Bau von etwa 250 zusätzlichen kleinen Wasserkraftanlagen.

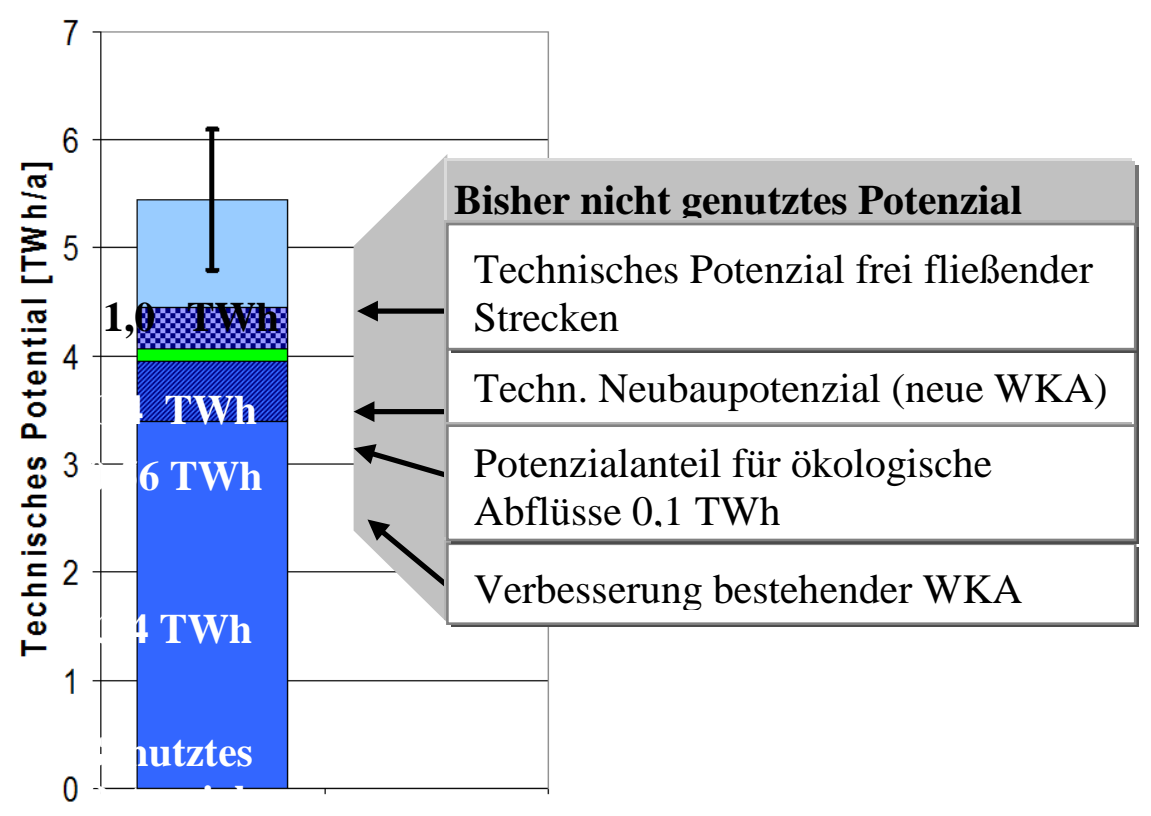

Abbildung 6 Aufteilung des technischen Potenzials an mittelgroßen und kleinen Gewässern

\section{Zusammenfassung}

Als Ausgangspunkt der Untersuchung wurden die in Betrieb befindlichen Wasserkraftanlagen in Deutschland ermittelt. In 2007 waren das etwa 7.400. Bei einer Gesamtleistung von ca. 4,05 GW beträgt der langjährige Mittelwert des genutzten Wasserkraftpotenzials etwa 20,9 TWh (die ausländischen Anteile der Wasserkraftanlagen an deutschen Grenzgewässern und die Daten der rein für die Eigenversorgung arbeitenden WKA sind hierin nicht enthalten).

Das Zubaupotenzial wurde ausgehend vom theoretischen Linienpotenzial der deutschen Gewässer unter Berücksichtigung der Fließverluste und unter Einbeziehung der realen Wirkungs- und Ausbaugrade von Wasserkraftanlagen abgeleitet. Als Ergebnis der Untersuchung wird das theoretische Linienpotenzial für Deutschland auf 92,6 TWh und das daraus abgeleitete technische Potenzial auf 33,2 bis 42,1 TWh beziffert. 
Das ermittelte technische Potenzial ist das Ergebnis einer rein physikalischen bzw. technischen Untersuchung. Es stellt das theoretisch maximal nutzbare Potenzial und damit die Obergrenze des nutzbaren Wasserkraftpotenzials dar. Nach Abzug des genutzten Potenzials ergibt sich ein nicht genutztes technisches Potenzial von etwa 12,3 bis 21,2 TWh. Seine Realisierbarkeit wurde differenziert für große und mittelgroße und kleine Gewässer betrachtet.

Für die großen Gewässer, die vorwiegend über Wasserkraftanlagen der Leistung $\mathrm{P} \geq 1 \mathrm{MW}$ verfügen, leitet sich das technische Zubaupotenzial folgendermaßen $\mathrm{ab}$ :

- An Elbe, Oder, Donau und Rhein existieren große frei fließende Strecken, die einen erheblichen Anteil des ermittelten technischen Zubaupotenzials beinhalten. Geht man davon aus, dass diese frei fließenden Strecken erhalten bleiben sollen, reduziert sich das technische Zubaupotenzial an den großen Gewässern auf im Mittel 4,0 TWh.

- Der größte Anteil dieses Zubaupotenzials kann mit 2,55 TWh an bestehenden Standorten von Wasserkraftanlagen der Leistung $\geq 1$ MW durch technische Verbesserungen, durch Erhöhung des Anlagenwirkungsgrades und des Ausbaugrades realisiert werden.

- Die Errichtung von neuen WKA mit $\mathrm{P} \geq 1 \mathrm{MW}$ an bestehenden Querbauwerken führt zu eine zusätzlichen jährlichen Erzeugung von etwa $0,12 \mathrm{TWh}$.

An den großen Gewässern können somit ca. 2,7 TWh des verbleibenden Zubaupotenzials von 4,0 TWh durch den Umbau bestehender Standorte genutzt werden. Die verbleibenden 1,3 TWh könnten nur durch den Neubau von Staustufen mit Wasserkraftanlagen in bisher ungenutzten Gewässerstrecken realisiert werden. Derartige Neubauten werden jedoch wegen der bestehenden Randbedingungen, Nutzungen und Restriktionen als eher unwahrscheinlich eingeschätzt.

An mittelgroßen und kleinen Gewässern konnte ein technisch-ökologischökonomisches Zubaupotenzial von etwa 0,4 TWh entsprechend einem weiteren Ausbau um $18 \%$ ermittelt werden.

In den nächsten Jahrzehnten stehen aufgrund ablaufender Konzessionen vermehrt Neugenehmigungen von Wasserkraftanlagen an. Es ist zu erwarten, dass die bisher nur in begrenztem Umfang erforderlichen Modernisierungen dann vermehrt durchgeführt werden. Damit besteht die Chance, dass die neuesten technischen und ökologischen Standards berücksichtigt werden. 


\section{Literatur}

[1] Bundesministerium für Umwelt, Naturschutz und Reaktorsicherheit (BMU): Erneuerbare Energien in Zahlen, 6/2010.

[2] Keller, H.; Ruprecht, C.; Vogel, Fr. : Die Wasserkräfte des Berg- und Hügellandes in Preußen und benachbarten Staatsgebieten ("Preußenstudie"). Studie im Auftrag der Preußischen Herren Minister der öffentlichen Arbeiten, für Handel und Gewerbe und für Landwirtschaft, Domänen und Forsten, 1914.

[3] Frohnholzer, J.: Systematik der Wasserkräfte in der BRD, 1962. In:

Selbstverlag Bayerischen Wasserkraftwerke AG, München, 1963.

[4] Hildebrand, H.; Kern, K.: Ermittlung des Wasserkraftpotenzials von BadenWürttemberg. Gutachten des Institutes für Wasserbau und Kulturtechnik der Universität Karlsruhe, 1986.

[5] Bayerisches Staatsministerium für Landesentwicklung und Umweltfragen (Hrsg.): Bericht über den weiteren Ausbau der Wasserkraftnutzung in Bayern, 1995.

[6] Wagner, E.; Rindelhardt, U.: Stromgewinnung aus regenerativer Wasserkraft in Deutschland - Überblick. In: ew 106 (2007), Heft 25-26.

[7] Heimerl, S.; Giesecke, J.: Wasserkraftanteil an der elektrischen Stromerzeugung in Deutschland 2003. In: Wasserwirtschaft 94 (2004), Heft 10.

[8] Rindelhardt, U.: Wasserkraftnutzung in Ostdeutschland. In: Wasserwirtschaft 97 (2007), Heft 6, S. 33-36.

[9] Anderer, P.; Dumont, U.; Linnenweber, Ch.; Schneider, B.: Das Wasserkraftpotenzial in RLP, Korrespondenz Wasserwirtschaft (2009), Heft 4. [10] Anderer, P.; Dumont, U.; Kolf, R.: Das Wasserkraftpotenzial in NordrheinWestfalen, In: Wasser und Abfall (2007), Heft 7-8.

[11] DLM1000W: Bundesamt für Kartographie und Geodäsie.

[12] Anderer, P.; Dumont, U.; Heimerl, S.; Ruprecht, A.; Wolf-Schumann, U.: Das Wasserkraftpotenzial in Deutschland. In: WasserWirtschaft 100 (2010, Nr. 9,S. 12-16.

[13] Anderer, P.; Dumont, Stark, B.; Wolf-Schumann, U.: Vom Linienpotenzial zum technischen Potenzial - Methode. In: WasserWirtschaft 100 (2010), Nr. 9, S. 17-22.

[14] Bauer, N. ; Ruprecht, A.; Heimerl, S.: Ermittlung des Wasserkraftpotenzials an Wasserkraftanlagenstandorten mit einer Leistung über $1 \mathrm{MW}$ in Deutschland. In: WasserWirtschaft 100 (2010), Nr. 9,S. 23-27. 